Functional Analysis and Its Applications, Vol. 39, No. 4, pp. 271-283, 2005

Translated from Funktsional'nyi Analiz i Ego Prilozheniya, Vol. 39, No. 4, pp. 32-47, 2005

Original Russian Text Copyright (C) by V. V. Kozlov

\title{
Restrictions of Quadratic Forms to Lagrangian Planes, Quadratic Matrix Equations, and Gyroscopic Stabilization*
}

\author{
V. V. Kozlov \\ Received June 24, 2005
}

ABSTRACT. We discuss the symplectic geometry of linear Hamiltonian systems with nondegenerate Hamiltonians. These systems can be reduced to linear second-order differential equations characteristic of linear oscillation theory. This reduction is related to the problem on the signatures of restrictions of quadratic forms to Lagrangian planes. We study vortex symplectic planes invariant with respect to linear Hamiltonian systems. These planes are determined by the solutions of quadratic matrix equations of a special form. New conditions for gyroscopic stabilization are found.

KEY WORDS: Hamiltonian function, symplectic structure, quadratic form, Williamson normal form, vortex plane.

In this paper, we discuss various aspects of the theory of linear Hamiltonian systems with nondegenerate Hamiltonian given by a quadratic form in a $2 n$-dimensional phase space. In Sec. 1 , the possibility of reducing general Hamiltonian systems to a second-order differential equation in an $n$-dimensional space is established, which is characteristic of the theory of small oscillations in mechanical systems. This reduction is related to the solution of the following problem: find an $n$-dimensional Lagrangian plane such that the restriction of the Hamiltonian function to this plane is a nondegenerate quadratic form. We also discuss the more complicated problem on the possible signatures of the restrictions of a quadratic form to Lagrangian planes. In particular, we establish that (just as in mechanical problems) the inertia matrix of the reduced second-order system can be made positive definite.

The terms linear in velocities correspond to the so-called gyroscopic forces that do not affect the conservation of total energy. Their structure is determined by a skew-symmetric operator $\Gamma$ in an $n$-dimensional space. This operator is assumed to be nondegenerate. In particular, the number $n$ of degrees of freedom is even. The classical gyroscopic stabilization problem is related to the study of conditions on the structure of $\Gamma$ under which the equilibrium of the Hamiltonian system becomes stable. Despite numerous results in this direction, the gyroscopic stabilization problem is far from being comprehensively solved yet. We consider the stability problem for small values of the norm $\left\|\Gamma^{-1}\right\|$.

Our approach involves seeking invariant $n$-dimensional planes $\Sigma$ of the Hamiltonian system such that $\Sigma$ itself is a symplectic space (see Sec. 2.) The symplectic structure on $\Sigma$ is defined as the restriction to $\Sigma$ of the original symplectic structure of the phase space. These invariant planes can be referred to as vortex or Cartesian planes (for the reasons given in [1]). The invariant planes are found as solutions of a quadratic matrix equation of a special form. The solutions that correspond to vortex planes come in pairs. Furthermore,

- the original $2 n$-dimensional phase space splits into the direct sum of $n$-dimensional vortex planes $\Sigma_{1}$ and $\Sigma_{2}$;

- $\Sigma_{1}$ and $\Sigma_{2}$ bear Hamiltonian systems with $n / 2$ degrees of freedom;

- the Hamiltonians of these systems are the restrictions of the original Hamiltonian to $\Sigma_{1}$ and $\Sigma_{2}$;

* This research was performed in the framework of the Program "State Support for Leading Scientific Schools" (grant No. NSh-136.2003.1). 
- the equilibrium position of the original Hamiltonian system is stable if and only if the equilibrium position of each of these Hamiltonian systems with half as many degrees freedom is stable.

According to Alan Weinstein's "main principle of symplectic geometry," all objects of interest in this geometry are Lagrangian manifolds. However, the author of the present paper sticks to another principle, stated in [1]: it is useful to study vortex manifolds, especially from the viewpoint of stability theory, since the restriction of the Hamiltonian to an $n$-dimensional invariant vortex manifold is a nonconstant function, which can be used as a Lyapunov function in some cases.

In Sec. 3, the quadratic matrix equation is solved with the help of the contraction mapping method. Under certain conditions, the solution with maximum norm generates a stable Hamiltonian system with $n / 2$ degrees of freedom. Thus the stability problem is reduced to the study of an additional linear Hamiltonian system with half as many degrees of freedom. In Sec. 4, we solve this matrix equation by expanding the solutions into power series with respect to an auxiliary parameter. The convergence of these series is proved with the use of a majorant series whose coefficients are the Catalan numbers, well known in combinatorics.

In Sec. 5, we describe the structure of invariant vortex planes for Hamiltonian systems with a simple spectrum.

The author is grateful to S. V. Bolotin, D. V. Treshchev, and A. A. Shkalikov for valuable discussions.

\section{Reduction of Linear Hamiltonian Systems}

Consider the second-order linear differential equation

$$
M \ddot{x}+\Gamma \dot{x}+P x=0, \quad x \in \mathbb{R}^{n} .
$$

Here $M,|M| \neq 0$, and $P,|P| \neq 0$, are symmetric matrices, and $\Gamma$ is a skew-symmetric matrix, $\Gamma^{T}=-\Gamma$. As usual, the dot stands for the time derivative. If $M$ is positive definite, then (1.1) describes small oscillations of a mechanical system with kinetic energy $(M \dot{x}, \dot{x}) / 2$ and potential energy $(P x, x) / 2$. The bracket $($,$) denotes the standard inner product in \mathbb{R}^{n},(u, v)=\sum_{1}^{n} u_{j} v_{j}$. The term $-\Gamma \dot{x}$ has the meaning of additional gyroscopic forces.

In the general case, Eq. (1.1) has the energy integral

$$
H=\frac{1}{2}(M \dot{x}, \dot{x})+\frac{1}{2}(P x, x) .
$$

Clearly, Eq. (1.1) is Hamiltonian. Let us introduce the canonical conjugate momenta

$$
y=M \dot{x}+\Gamma x / 2
$$

and take the quadratic form (1.2) expressed in the variables $x$ and $y$ as the Hamiltonian function. Then we can pass from the second-order equation (1.1) to the Hamiltonian differential equations

$$
\dot{x}=\frac{\partial H}{\partial y}, \quad \dot{y}=-\frac{\partial H}{\partial x} .
$$

Conversely, let

$$
\dot{v}=\frac{\partial H}{\partial u}, \quad \dot{u}=-\frac{\partial H}{\partial v}, \quad u, v \in \mathbb{R}^{n},
$$

be a linear Hamiltonian system with quadratic Hamiltonian

$$
H=\frac{1}{2}(A u, u)+(B u, v)+\frac{1}{2}(C v, v),
$$

and let, say, the symmetric matrix $A$ be nonsingular. Then the first equation in system (1.3) can be solved for $u$,

$$
u=A^{-1} \dot{v}-A^{-1} B^{T} v .
$$


The substitution of this expression into the second equation in (1.3) results in the second-order differential equation

$$
A^{-1} \ddot{v}+\left(B A^{-1}-A^{-1} B^{T}\right) \dot{v}+\left(C-B A^{-1} B^{T}\right) v=0
$$

of the form (1.1). It is clear that $B A^{-1}-A^{-1} B^{T}$ is a skew-symmetric matrix and $C-B A^{-1} B^{T}$ is a symmetric matrix.

Theorem 1. For a linear Hamiltonian system with nondegenerate quadratic Hamiltonian $H$, there exist canonical variables $u$ and $v$ such that $|A| \neq 0$ in these variables.

This assertion is equivalent to the following.

Theorem 2. Let $H$ be a nondegenerate quadratic form in a $2 n$-dimensional symplectic vector space. Then there exists an $n$-dimensional Lagrangian plane $\Lambda$ such that the restriction of $H$ to $\Lambda$ is a nondegenerate quadratic form.

Indeed, if $|A| \neq 0$, then we can set $\Lambda=\{v=0\}$. Conversely, let $\Lambda$ be an $n$-dimensional Lagrangian plane in Theorem 2 , and let $u=\left(u_{1}, \ldots, u_{n}\right)$ be Cartesian coordinates on $\Lambda$. These coordinates can always be extended to canonical variables $u_{1}, \ldots, u_{n}, v_{1}, \ldots, v_{n}$ in the entire symplectic space. In these variables, we have $\Lambda=\{v=0\}, H$ has the form (1.4), and, obviously, $|A| \neq 0$.

There is no short proof of Theorem 2 known to the author. The most straightforward way is to use Williamson's theory of normal forms [2]. According to this approach, the real symplectic space on which the quadratic Hamiltonian $H$ is defined splits into a direct sum of pairwise skew-orthogonal real symplectic subspaces such that $H$ can be represented as the sum of partial Hamiltonians of special form defined on these subspaces. This reduces the problem to finding Lagrangian planes in the symplectic subspaces for the nondegenerate partial Hamiltonians. The desired $n$-dimensional Lagrangian plane is the direct sum of these "partial" Lagrangian planes.

According to Williamson, form

(a) the partial Hamiltonian corresponding to a real pair $\pm a$ of eigenvalues of order $k$ has the

$$
H=-a \sum_{j=1}^{k} p_{j} q_{j}+\sum_{j=1}^{k-1} p_{j} q_{j+1}
$$

(b) the partial Hamiltonian corresponding to a quadruple $\pm a \pm i b$ of order $k$ has the form

$$
H=-a \sum_{j=1}^{2 k} p_{j} q_{j}+b \sum_{j=1}^{k}\left(p_{2 j-1} q_{2 j}-p_{2 j} q_{2 j-1}\right)+\sum_{j=1}^{2 k-2} p_{j} q_{j+2}
$$
form

(c) the partial Hamiltonian corresponding to a pure imaginary pair $\pm i b$ of order $2 k+1$ has the

$$
H= \pm \frac{1}{2}\left[\sum_{j=1}^{k}\left(b^{2} p_{2 j} p_{2 k-2 j+2}+q_{2 j} q_{2 k-2 j+2}\right)-\sum_{j=1}^{k+1}\left(b^{2} p_{2 j-1} p_{2 k-2 j+3}+q_{2 j-1} q_{2 k-2 j+3}\right)\right]-\sum_{j=1}^{2 k} p_{j} q_{j+1} ;
$$
form

(d) the partial Hamiltonian corresponding to a pure imaginary pair $\pm i b$ of order $2 k$ has the

$$
\begin{aligned}
H= \pm \frac{1}{2}[ & \sum_{j=1}^{k}\left(b^{-2} q_{2 j-1} q_{2 k-2 j+1}+q_{2 j} q_{2 k-2 j+2}\right) \\
& \left.-\sum_{j=1}^{k-1}\left(b^{2} p_{2 j+1} p_{2 k-2 j+1}+p_{2 j+2} p_{2 k-2 j+2}\right)\right]-b^{2} \sum_{j=1}^{k} p_{2 j-1} q_{2 j}+\sum_{j=1}^{k} p_{2 j} q_{2 j-1} .
\end{aligned}
$$

In cases (a) and (b), the partial Hamiltonians have the form

$$
H=(B p, q) \text {. }
$$

The following simple lemma holds. 
Lemma 1. Let $\lambda_{1}, \ldots, \lambda_{n}$ be the eigenvalues of the $n \times n$ matrix $B$, and suppose that

$$
\lambda_{j}+\lambda_{k} \neq 0
$$

for all $j$ and $k$. Then for arbitrary nonnegative integers $i^{+}$and $i^{-}, i^{+}+i^{-}=n$, there exists a Lagrangian plane $\Lambda$ such that the signature of the quadratic form $\left.H\right|_{\Lambda}$ is $\left(i^{+}, i^{-}\right)$.

In particular, $\left.H\right|_{\Lambda}$ is nondegenerate.

Proof. We shall seek $\Lambda$ in the form

$$
p=R q, \quad R^{T}=R .
$$

Let $h=(Q q, q) / 2$ be an arbitrary quadratic form with signature $\left(i^{+}, i^{-}\right)$. We set $\left.H\right|_{\Lambda}=h$. This is equivalent to the linear matrix equation

$$
B R+R B^{T}=Q
$$

By virtue of condition (1.5), this equation is always solvable for an arbitrary symmetric matrix $Q$ (say, see [3]), as desired.

In case (a), the matrix $B$ has $k$ eigenvalues equal to $-a$, and in case (b), there are $2 k$ eigenvalues of $B$ equal to $-a \pm i b$. Since $a \neq 0$, condition (1.5) necessarily holds. This proves Theorem 2 for cases (a) and (b).

We set $\Lambda=\{p=0\}$ in the remaining cases. Then

$$
\left.H\right|_{\Lambda}=\frac{1}{2}(C q, q)
$$

and (as can readily be verified) $\operatorname{det} C \neq 0$, as desired.

If a quadratic form $H$ in a $2 n$-dimensional space in nondegenerate, then its restriction to some $n$-dimensional (not necessarily Lagrangian) plane is a positive or negative definite quadratic form. The question is whether this conclusion is true for Lagrangian planes. If the answer were "yes," it would be possible to reduce an arbitrary linear Hamiltonian system with nondegenerate Hamiltonian to the linear mechanical system (1.1) with positive definite inertia matrix $M$.

However, the answer is "no." The quadratic form

$$
H=u_{1}^{2}+v_{1}^{2}-u_{2}^{2}-v_{2}^{2}
$$

can serve as a simple counterexample. Indeed, consider the Lagrangian plane $\Lambda$ given by the equations

$$
u_{1}=\alpha v_{1}+\beta v_{2}, \quad u_{2}=\beta v_{1}+\gamma v_{2} .
$$

The restriction of the form (1.6) to $\Lambda$ is

$$
\left(\alpha^{2}-\beta^{2}+1\right) v_{1}^{2}+2 \beta(\alpha-\gamma) v_{1} v_{2}+\left(\beta^{2}-\gamma^{2}-1\right) v_{2}^{2} .
$$

The determinant of the matrix of this quadratic form is

$$
\Delta=-\left(\beta^{2}-\alpha \gamma\right)^{2}+2 \beta^{2}-\alpha^{2}-\gamma^{2}-1 .
$$

This function of the three variables $\alpha, \beta$, and $\gamma$ attains its maximum on the curve $\alpha=\gamma=\omega$, $\beta= \pm \sqrt{1-\omega^{2}}(\omega \in \mathbb{R})$, and this maximum is zero. Consequently, $\Delta$ cannot be positive, and therefore, the quadratic form (1.8) cannot be sign definite.

The cases in which the projection of $\Lambda$ onto the coordinate $\left(v_{1}, v_{2}\right)$-plane is not one-to-one are described by the equations

$$
u_{1}=\alpha v_{1}+\beta u_{2}, \quad v_{2}=-\beta v_{1}
$$

or

$$
u_{2}=\alpha v_{2}+\beta u_{1}, \quad v_{1}=-\beta v_{2} .
$$

It is quite easily seen that the restriction of (1.6) to the Lagrangian planes (1.9) and (1.10) is never a sign definite quadratic form. 
Nevertheless, the Hamiltonian equations with Hamiltonian (1.6) can be reduced to a secondorder system of the form (1.1) with positive definite matrix $M$,

$$
\ddot{v}_{1}+v_{1}=0, \quad \ddot{v}_{2}+v_{2}=0 .
$$

However, this is no contradiction at all, since the canonical variables $u_{1}, v_{1}$ and $u_{2}, v_{2}$ in the Hamiltonian (1.6) separate. These observations can be generalized as is shown below.

Let

$$
\mathbb{R}^{2 n}=\Pi_{1} \oplus \cdots \oplus \Pi_{m}
$$

let $\Pi_{k}$ and $\Pi_{l}(k \neq l)$ be skew-orthogonal symplectic subspaces, let $H_{l}: \Pi_{l} \rightarrow \mathbb{R}$ be partial Hamiltonians, and let

$$
H=H_{1}+\cdots+H_{m} .
$$

Furthermore, let $\left(i_{l}^{+}, i_{l}^{-}\right)$be the signature of the quadratic form $H_{l}$. In view of the nondegeneracy assumption, the sum $i_{l}^{+}+i_{l}^{-}$is equal to $\operatorname{dim} \Pi_{l}$ and hence is even.

Theorem 3. Let $j_{l}^{+}$and $j_{l}^{-}$be nonnegative integers such that

$$
j_{l}^{+} \leqslant i_{l}^{+}, \quad j_{l}^{-} \leqslant i_{l}^{-}, \quad j_{l}^{+}+j_{l}^{-}=\left(i_{l}^{+}+i_{l}^{-}\right) / 2 .
$$

Then there exists an $n$-dimensional Lagrangian plane $\Lambda$ such that the signature of the quadratic form $\left.H\right|_{\Lambda}$ is $\left(j^{+}, j^{-}\right)$, where $j^{ \pm}=j_{1}^{ \pm}+\cdots+j_{m}^{ \pm}$.

For quadratic form (1.6), we have $m=2$, and the signatures of the partial Hamiltonians $H_{1}$ and $H_{2}$ are $(2,0)$ and $(0,2)$, respectively. Hence, by Theorem 3 , the signature of a nondegenerate restriction of the quadratic form (1.6) to a Lagrangian plane can only be $(1,1)$.

In the typical case where the spectrum of the linear Hamiltonian system with Hamiltonian $H$ is simple, the proof of Theorem 3 is extremely easy. For partial Hamiltonians with nontrivial Jordan blocks of the form (a) and (b), Theorem 3 follows from Lemma 1. In cases (c) and (d), the conclusion of the theorem can be verified by cumbersome calculations.

The signatures of the partial Hamiltonians were calculated in [4]. In case (a), the signature is $(k, k)$, in case (b), it is $(2 k, 2 k)$, in case (c), it is either $(2 k+2,2 k)$ or $(2 k, 2 k+2)$, and in case $(\mathrm{d})$, we once more have the signature $(2 k, 2 k)$.

Corollary 1. A linear Hamiltonian system with $n$ degrees of freedom and nondegenerate Hamiltonian can be reduced to the second-order differential equation

$$
\ddot{x}+\Gamma \dot{x}+P x=0, \quad x \in \mathbb{R}^{n},
$$

where $\Gamma^{T}=-\Gamma, P^{T}=P$, and $|P| \neq 0$.

To prove this assertion, we use (1.11) and (1.12). By Theorems 1 and 3, the Hamiltonian equations are reduced to Eq. (1.1) with

$$
M=\operatorname{diag}\left(M_{1}, \ldots, M_{n}\right), \quad \Gamma=\operatorname{diag}\left(\Gamma_{1}, \ldots, \Gamma_{m}\right), \quad P=\operatorname{diag}\left(P_{1}, \ldots, P_{m}\right),
$$

where the symmetric matrices $M_{j}$ are either positive or negative definite. If $M_{j}$ is negative definite, then the signs of $M_{j}, \Gamma_{j}$, and $P_{j}$ should be changed. As a result, Eq. (1.1) retains its form, and the matrix $M$ becomes positive definite. After that, Eq. (1.1) is brought to the form (1.13) by a standard trick.

Corollary 2. A linear Hamiltonian system with simple spectrum can be reduced to Eq. (1.13) with a skew-symmetric matrix $\Gamma$ whose rank is twice the number of complex quadruples in the spectrum.

One should not have the impression that for $|\Gamma| \neq 0$ the simple spectrum of Eq. (1.13) consists of complex quadruples alone.

Corollary 3. A linear system

$$
\dot{x}=A x, \quad x \in \mathbb{R}^{s},
$$

that has the only equilibrium position $x=0(|A| \neq 0)$ and admits a nondegenerate quadratic first integral can always be reduced to the form (1.13). 
Indeed, under these conditions system (1.14) is Hamiltonian [5]. In particular, $s$ is even $(s=2 n)$. It remains to use Corollary 1.

The reduction of Hamiltonian systems to the form (1.13) is of fundamental character, but it is not fully constructive. In what follows, we everywhere study Eq. (1.1) without assuming that the matrix $M$ is positive definite.

\section{Invariant Vortex Planes}

Let

$$
\Sigma=\{x, \dot{x}: \dot{x}=A x\}
$$

be an invariant $n$-dimensional plane for system (1.1); i.e., if a phase trajectory of system (1.1) meets $\Sigma$, then it lies entirely in $\Sigma$. The invariance condition for $\Sigma$ is expressed by the following quadratic equation for the matrix $A$ :

$$
(M A+\Gamma) A+P=0 .
$$

We set

$$
A=M^{-1}(D-\Gamma / 2) .
$$

Then the matrix $D$ satisfies the quadratic equation

$$
(D+\Gamma / 2) M^{-1}(D-\Gamma / 2)+P=0 .
$$

It is clear that

$$
\left(D^{T}+\Gamma / 2\right) M^{-1}\left(D^{T}-\Gamma / 2\right)+P=0 .
$$

Consequently,

also satisfies Eq. (2.1).

$$
A^{\prime}=M^{-1}\left(D^{T}-\Gamma / 2\right)
$$

The matrices $A$ and $A^{\prime}$ are related by the formula

$$
A^{\prime}=M^{-1} A^{T} M-M^{-1} \Gamma .
$$

This is an analog of Viete's theorem for the matrix equation (2.1). If $\Sigma$ is invariant, then the plane $\Sigma^{\prime}=\left\{\dot{x}=A^{\prime} x\right\}$ is also invariant. In accordance with this remark, it is natural to consider the following two linear differential equations:

$$
\dot{x}=A x, \quad x \in \mathbb{R}^{n},
$$

and

$$
\dot{x}=A^{\prime} x, \quad x \in \mathbb{R}^{n} .
$$

In the canonical variables $x, y$, the invariant plane $\{\dot{x}=A x\}$ has the form $\{y=D x\}$. If $D$ is a symmetric matrix, then this is a Lagrangian plane. We shall consider another extreme case in which the matrix $\operatorname{rot}(D x)=D-D^{T}$ is nonsingular. Such invariant planes can naturally be called vortex planes [1].

Theorem 4. If $\left|D-D^{T}\right| \neq 0$, then

(1) the phase space $\mathbb{R}^{2 n}=\{x, \dot{x}\}$ is the direct sum of the $n$-dimensional planes $\Sigma$ and $\Sigma^{\prime}$;

(2) the equilibrium position $x=0, \dot{x}=0$ of system (1.1) is stable if and only if all solutions of the linear differential equations (2.5) and (2.6) are bounded.

Proof. If the $n$-dimensional planes $\Sigma$ and $\Sigma^{\prime}$ have a common point $(x, \dot{x}) \neq(0,0)$, then $A x=A^{\prime} x$ for some $x \neq 0$. Therefore, by (2.2) and (2.4),

$$
\left|A-A^{\prime}\right|=\left|M^{-1}\right|\left|D-D^{T}\right|=0,
$$

and consequently, the skew-symmetric matrix $D-D^{T}$ is singular. Therefore, $\mathbb{R}^{2 n}=\Sigma \oplus \Sigma^{\prime}$. Each of the linear systems (2.2) and (2.3) has $n$ linearly independent solutions. By the already established property (1) and by the invariance of the planes $\Sigma$ and $\Sigma^{\prime}$, these $2 n$ solutions form a basis in the entire $2 n$-dimensional solution space of system (1.1), as desired.

We note that the skew-symmetric matrix $D-D^{T}$ can be nonsingular only for even $n$. 
Set

$$
\begin{gathered}
h(x)=\left.H\right|_{\dot{x}=A x}=\frac{1}{2}\left(A^{T} M A x, x\right)+\frac{1}{2}(P x, x), \\
h^{\prime}(x)=\left.H\right|_{\dot{x}=A^{\prime} x}=\frac{1}{2}\left(A^{\prime T} M A^{\prime} x, x\right)+\frac{1}{2}(P x, x) .
\end{gathered}
$$

It is clear that the quadratic forms $h$ and $h^{\prime}$ are first integrals of the linear systems (2.5) and (2.6), respectively.

Theorem 5. If $\left|D-D^{T}\right| \neq 0$, then the linear systems (2.5) and (2.6) are Hamiltonian with Hamiltonian functions $h$ and $h^{\prime}$, respectively.

Proof. Indeed,

$$
A^{T} M A=\left(D^{T}+\Gamma / 2\right) M^{-1}(D-\Gamma / 2)
$$

Using formulas (2.2) and (2.3), we obtain

$$
\begin{aligned}
-\partial h / \partial x & =\left[-\left(D^{T}+\Gamma / 2\right) M^{-1}(D-\Gamma / 2)-P\right] x \\
& =\left[-\left(D^{T}+\Gamma / 2\right) M^{-1}(D-\Gamma / 2)+(D+\Gamma / 2) M^{-1}(D-\Gamma / 2)\right] x \\
& =\left[\left(D-D^{T}\right) M^{-1}(D-\Gamma / 2)\right] x=\left(D-D^{T}\right) \dot{x} .
\end{aligned}
$$

For brevity, we set $D-D^{T}=\Omega$ and $h(x)=(B x, x) / 2$. Then the linear system $(2.5)$ acquires the form

$$
\Omega \dot{x}=-B x .
$$

We set $x=C z$, where the matrix $C$ satisfies the relation

$$
C^{T} \Omega C=J=\left(\begin{array}{cc}
0 & E \\
-E & 0
\end{array}\right)
$$

in which $E$ is the $n / 2 \times n / 2$ identity matrix. In the new variables, Eq. (2.7) has the form of a linear Hamiltonian system,

where

$$
J \dot{x}=-\frac{\partial h}{\partial z}
$$

as desired.

$$
h(z)=\left.\frac{1}{2}(B x, x)\right|_{x=C z}=\frac{1}{2}\left(C^{T} B C z, z\right),
$$

Thus, in view of Theorem 4, under the condition

$$
\left|D-D^{T}\right| \neq 0
$$

the stability problem for the linear Hamiltonian system (1.1) with $n$ degrees of freedom is reduced to the stability problem for two linear Hamiltonian systems with $n / 2$ degrees of freedom.

Theorem 6. If the Hamiltonian system (1.1) with $n$ degrees of freedom admits an invariant vortex plane, then the spectrum of (1.1) splits into two parts each of which consists of $n$ points and is invariant (counting multiplicities) under the reflections in the real and imaginary axes.

This is a consequence of Theorems 4 and 5 as well as of a well-known result on the spectrum of a linear Hamiltonian system.

\section{The Contraction Mapping Method and Invariant Vortex Planes}

The problem on the solutions of a quadratic matrix equation is of interest in itself. It is discussed in [6] as a new problem, although some special cases were already considered earlier. (For example, see $[1]$.

There are some known simple quadratic matrix equations that have a continuum of solutions (for example, $A^{2}=0$ ) and also equations that do not admit solutions (even with complex entries) at all [6]. Therefore, the existence conditions for matrix solutions and the study of properties of these solutions are topical. In [7], the existence conditions are discussed for solutions of Eq. (2.1) 
that have the form (2.2) with symmetric matrix $D$. These solutions generate Lagrangian invariant planes.

Lemma 2. The equation $M X^{2}+\Gamma X+P=0,|\Gamma| \neq 0$, has a solution if

$$
\left\|\Gamma^{-1} M\right\|\left\|\Gamma^{-1} P\right\| \leqslant 1 / 4
$$

moreover,

$$
\|X\| \leqslant \frac{1-\sqrt{1-4\left\|\Gamma^{-1} M\right\|\left\|\Gamma^{-1} P\right\|}}{2\left\|\Gamma^{-1} M\right\|} .
$$

Here $\|\cdot\|$ is an arbitrary matrix norm. If

$$
\left\|\Gamma^{-1} M\right\|\left\|\Gamma^{-1} P\right\|<1 / 4,
$$

then this equation has a unique solution satisfying condition (3.2).

Proof. In view of the assumption of invertibility for the matrix $\Gamma$, we rewrite the quadratic equation in the form

$$
X=F(X)=-\Gamma^{-1}\left(M X^{2}+P\right) .
$$

It can readily be verified that under condition (3.1) the nonlinear operator $F$ maps the ball (3.2) into itself. Consequently, the operator $F$ has a fixed point in this ball by the Bohl-Brouwer theorem.

Furthermore, for arbitrary $X$ and $Y$ in the ball (3.2) we have

$$
\begin{aligned}
\|F(X)-F(Y)\| & =\left\|-\Gamma^{-1} M\left(X^{2}-X Y+X Y-Y^{2}\right)\right\| \\
& \leqslant\left\|-\Gamma^{-1} M\right\|(\|X\|+\|Y\|)\|X-Y\| \\
& \leqslant\left(1-\sqrt{1-4\left\|\Gamma^{-1} M\right\|\left\|\Gamma^{-1} P\right\|}\right)\|X-Y\| .
\end{aligned}
$$

Thus if condition (3.3) is satisfied, then $F$ is a contraction mapping in the ball (3.2), as desired.

To simplify the further exposition, we use a linear transformation to bring the symmetric matrix $M$ to the diagonal form

$$
M=\operatorname{diag}( \pm 1, \ldots, \pm 1) .
$$

Setting $x=C z$, we rewrite Eq. (1.1) in the equivalent form

$$
C^{T} M C \ddot{z}+C^{T} \Gamma C \dot{z}+C^{T} P C z=0 .
$$

There exists a nonsingular matrix $C$ such that $C^{T} M C$ has the form (3.4); in this case, the matrix $C^{T} \Gamma C\left(C^{T} P C\right)$ obviously remains skew-symmetric (symmetric) and nonsingular.

In what follows, we shall use the matrix norms $\|\cdot\|$ in which $\|M\|=1$. They include all natural operator norms. For example, the two norms

$$
\|X\|_{1}=\max _{j} \sum_{k}\left|x_{j k}\right|, \quad\|X\|_{2}=\max _{k} \sum_{j}\left|x_{j k}\right|,
$$

where $X=\left(x_{j k}\right)$, satisfy this condition. In these cases, we have

$$
\|X M\|=\|M X\|=\|X\| \text {. }
$$

Theorem 7. If

$$
\left\|\Gamma^{-1}\right\|\left\|\Gamma^{-1} P\right\|<1 / 4
$$

then Eq. (1.1) has two invariant vortex planes.

Indeed, in view of (3.5), condition (3.6) is equivalent to (3.3). Hence the existence of two solutions of matrix equation (2.1) follows from Lemma 2. It remains to verify that the skewsymmetric matrix

is nonsingular. Indeed,

$$
D-D^{T}=M A-A^{T} M+\Gamma
$$

$$
\Gamma^{-1}\left(D-D^{T}\right)=\Gamma^{-1}\left(M A-A^{T} M\right)+E .
$$

If

$$
\left\|\Gamma^{-1}\left(M A-A^{T} M\right)\right\| \leqslant 2\left\|\Gamma^{-1}\right\|\|A\|<1,
$$


then, obviously, $\left|D-D^{T}\right| \neq 0$. Since

$$
1-\sqrt{1-x} \leqslant x, \quad 0 \leqslant x \leqslant 1,
$$

we see that inequality (3.2) provides the coarser estimate

$$
\|A\| \leqslant 2\left\|\Gamma^{-1} P\right\| \text {. }
$$

It remains to note that (3.6) and (3.8) imply (3.7).

Remark. Inequality (3.6) can be replaced by the coarser condition

$$
\sqrt{\|P\|}\left\|\Gamma^{-1}\right\|<1 / 2 \text {. }
$$

If $P$ is a negative definite matrix, then Bolotin's sufficient condition for the gyroscopic stabilization of the equilibrium position $x=0$ has the form (see [1])

$$
\left\|(-P)^{1 / 2}\right\|\left\|\Gamma^{-1}\right\|<1 / 2 .
$$

Now consider the case in which (as in mechanical problems) $M$ is the identity matrix.

Theorem 8. If

$$
\left\|\Gamma^{-1}\right\|\left\|\Gamma^{-1} P\right\|<2 / 9,
$$

then the quadratic form $h^{\prime}(x), x \in \mathbb{R}^{n}$, is positive definite.

Needless to say, (3.9) is a stronger condition than (3.6). However, these conditions are rather close, since $2 / 9$ differs from $1 / 4$ only by $1 / 36$.

Corollary 1. Suppose that condition (3.9) holds. Then the equilibrium position $x=0$ of system (1.1) is stable if and only if so is the equilibrium position of the Hamiltonian system (2.5) with half as many degrees of freedom.

We recall that the $n \times n$ matrix $A$ in (2.5) is defined as the unique solution of (2.1) satisfying (3.2).

Proof of Theorem 8. Since $M=E$, we have

$$
2 h^{\prime}=\left(A^{\prime} x, A^{\prime} x\right)+(P x, x), \quad A^{\prime}=A^{T}-\Gamma .
$$

Set $x=\Gamma^{-1} z$. Then (3.10) acquires the form

$$
2 h^{\prime}=(z, z)+(B z, z), \quad B=\Gamma^{-1} A-A^{T} \Gamma^{-1}-\Gamma^{-1} A A^{T} \Gamma^{-1}-\Gamma^{-1} P \Gamma^{-1} .
$$

It suffices to show that $\|B\|<1$. We estimate the norm of the matrix $B$ :

$$
\|B\| \leqslant 2\left\|\Gamma^{-1}\right\|\|A\|+\left(\left\|\Gamma^{-1}\right\|\|A\|\right)^{2}+\left\|\Gamma^{-1}\right\|\left\|\Gamma^{-1} P\right\| .
$$

By (3.2),

$$
\left\|\Gamma^{-1}\right\|\|A\| \leqslant \frac{1-\sqrt{1-4 \alpha}}{2}, \quad \alpha=\left\|\Gamma^{-1}\right\|\left\|\Gamma^{-1} P\right\| .
$$

Taking (3.11) into account, we obtain the inequality

$$
1-\sqrt{1-4 \alpha}+\frac{(1-\sqrt{1-4 \alpha})^{2}}{4}+\alpha<1,
$$

whose solution $\alpha<2 / 9$ is found readily. The proof is complete.

Corollary 2. If $M=E$ and condition (3.9) is satisfied, then the signature of the quadratic form $h(x), x \in \mathbb{R}^{n}$, coincides with that of the potential energy $(P x, x) / 2$.

In particular, let the potential energy assume the maximum value at the equilibrium position $x=0$. Then condition (3.9) ensures the gyroscopic stabilization of the equilibrium position of system (1.1). 


\section{Large Gyroscopic Forces}

It is also possible to represent the solutions of matrix equation (2.1) in the form of convergent series. To this end, we replace the matrix $\Gamma$ of gyroscopic forces by $N \Gamma$ and assume that the parameter $N$ is large (asymptotically, $N \rightarrow \infty$ ). We set $A=N X$ and $\varepsilon=1 / N^{2}$. Then the matrix equation (2.1) acquires the form

$$
(M X+\Gamma) X+\varepsilon P=0 .
$$

We seek the solution of Eq. (4.1) in the form of a series in powers of $\varepsilon$,

$$
X=X_{0}+\varepsilon X_{1}+\varepsilon^{2} X_{2}+\cdots .
$$

By substituting (4.2) into (4.1) and by matching the coefficients of like powers of $\varepsilon$, we obtain a chain of algebraic relations, from which the matrices $X_{0}, X_{1}, \ldots$ can be found successively. For $X_{0}$, we obtain the equation

$$
\left(M X_{0}+\Gamma\right) X_{0}=0
$$

which has two solutions, $X_{0}=-M^{-1} \Gamma$ and $X_{0}=0$.

For the second case, the remaining coefficients are found from the relations

$$
\begin{gathered}
\Gamma X_{1}+P=0, \quad \Gamma X_{2}+M X_{1}^{2}=0, \quad \Gamma X_{3}+M\left(X_{1} X_{2}+X_{2} X_{1}\right)=0, \\
\Gamma X_{4}+M\left(X_{1} X_{3}+X_{2}^{2}+X_{3} X_{1}\right)=0, \quad \ldots,
\end{gathered}
$$

whence we successively obtain

$$
X_{1}=-\Gamma^{-1} P, \quad X_{2}=-\Gamma^{-1} M\left(\Gamma^{-1} P\right)^{2}, \quad \ldots .
$$

To find convergence conditions for the series (4.2), we estimate the norms of the matrix coefficients. Setting

we successively obtain

$$
U=\Gamma^{-1} P, \quad V=\Gamma^{-1} M,
$$

$$
\begin{gathered}
\left\|X_{1}\right\|=\|U\|, \quad\left\|X_{2}\right\| \leqslant\|V\|\|U\|^{2}, \quad\left\|X_{3}\right\| \leqslant 2\|V\|^{2}\|U\|^{3}, \\
\left\|X_{4}\right\| \leqslant\|V\|\left(4\|U\|\|V\|^{2}\|U\|^{3}+\|V\|^{2}\|U\|^{4}\right)=5\|V\|^{3}\|U\|^{4}, \\
\left\|X_{5}\right\| \leqslant 14\|V\|^{4}\|U\|^{5}, \quad \ldots
\end{gathered}
$$

Thus

$$
\left\|X_{k}\right\| \leqslant æ_{k}\|V\|^{k-1}\|U\|^{k} .
$$

Relations (4.3) imply the recursion rule for calculating the coefficients $æ_{k}$,

$$
æ_{k+1}=æ_{1} æ_{k}+æ_{2} æ_{k-1}+\cdots+æ_{k-1} æ_{2}+æ_{k} æ_{1}, \quad æ_{1}=1 .
$$

We see that the $æ_{k}$ are the Catalan numbers, well known in combinatorics. Their generating function $\sum_{k=1}^{\infty} æ_{k} z^{k}$ is equal to $(1-\sqrt{1-4 z}) / 2$.

Consequently,

$$
\|X\| \leqslant \sum_{k=1}^{\infty} \varepsilon^{k}\left\|X_{k}\right\| \leqslant\|V\|^{-1} \sum_{k=1}^{\infty} \varepsilon^{k} æ_{k}(\|U\|\|V\|)^{k}=\frac{1-\sqrt{1-4 \varepsilon\|U\|\|V\|}}{2\|V\|} .
$$

The series (4.2) is obviously convergent under condition (3.3):

$$
\varepsilon\|U\|\|V\|=\left\|(N \Gamma)^{-1} M\right\|\left\|(N \Gamma)^{-1} P\right\|<1 / 4 .
$$

Since $A=N X$, it follows that the estimate (4.5) coincides with inequality (3.2) with $\Gamma$ replaced by $N \Gamma$.

Equation (4.1) has one more solution

$$
Y=-M^{-1} \Gamma+\varepsilon Y_{1}+\varepsilon^{2} Y_{2}+\cdots,
$$

which is also convergent under condition (4.6). These two solutions correspond to the two different vortex planes for the linear Hamiltonian system (1.1). 
The above result can be interpreted as follows: under condition (3.3), the series $\sum_{1}^{\infty} X_{k}$ converges to the solution of (2.1) satisfying (3.2).

For small $\varepsilon>0$, the Hamiltonian differential equations (2.5) and (2.6) have the form

$$
\dot{x}=-\frac{1}{\sqrt{\varepsilon}} M^{-1} \Gamma x+O(\sqrt{\varepsilon}), \quad \dot{x}=-\sqrt{\varepsilon} \Gamma^{-1} P x+O\left(\varepsilon^{3 / 2}\right) .
$$

Their Hamiltonians are the quadratic forms

$$
-\frac{1}{2 \varepsilon}\left(\Gamma M^{-1} \Gamma x, x\right)+\frac{1}{2}(P x, x)+O(\varepsilon), \quad \frac{1}{2}(P x, x)-\frac{\varepsilon}{2}\left(P \Gamma^{-1} M \Gamma^{-1} P x, x\right)+O(\varepsilon) .
$$

As $\varepsilon \rightarrow 0$, the signatures of these forms coincide with those of the quadratic forms

$$
-\left(\Gamma M^{-1} \Gamma x, x\right) \text { and }(P x, x),
$$

respectively.

In particular, let $M$ be a positive definite matrix (as in mechanical problems), and let the potential energy have a strict maximum at the equilibrium position $x=0$. Then the first quadratic form in (4.8) is positive definite and the second is negative definite. Consequently, in this case the equilibrium position is stable for large $N$, which is a well-known result on gyroscopic stabilization.

Thus for large $N$ the stability problem is reduced to the study of the second equation in (4.7),

$$
\Gamma \dot{x}=-P x,
$$

which is obviously of Hamiltonian nature.

\section{The Structure of Invariant Vortex Planes}

We shall briefly discuss the existence conditions for invariant vortex planes and the structure of these planes for the general Hamiltonian linear system

$$
J \dot{z}=-B z, \quad z \in \mathbb{R}^{2 n},
$$

where $J=-J^{-1}$ is the symplectic unit matrix and $B$ is a nonsingular symmetric matrix, which generates the Hamiltonian $H=(B z, z) / 2$. The vector space $\mathbb{R}^{2 n}$ has the natural symplectic structure, namely, the nondegenerate 2 -form

$$
\omega\left(z^{\prime}, z^{\prime \prime}\right)=\left(J z^{\prime}, z^{\prime \prime}\right), \quad \omega\left(z^{\prime}, z^{\prime \prime}\right)=-\omega\left(z^{\prime \prime}, z^{\prime}\right) .
$$

Generalizing the definition in Sec. 2, we refer to a plane $\Sigma$ passing through the origin as a vortex plane if the restriction $\widetilde{\omega}=\left.\omega\right|_{\Sigma}$ is a nondegenerate 2 -form. (For an arbitrary nonzero $x \in \Sigma$, there exists a vector $y \in \Sigma$ such that $\widetilde{\omega}(x, y) \neq 0$.) In particular, $n$ is even, and the pair $(\Sigma, \widetilde{\omega})$ itself is an $n$-dimensional symplectic space.

Let $\Sigma_{1}$ be an $n$-dimensional (not necessarily vortex) plane, and let $\Sigma_{2}$ be the skew-orthogonal complement of $\Sigma_{1}$,

$$
\Sigma_{2}=\left\{z \in \mathbb{R}^{2 n}: \omega(z, x)=0 \text { for all } x \in \Sigma_{1}\right\} .
$$

Since $J$ is nondegenerate, we have $\operatorname{dim} \Sigma_{2}=n$. The planes $\Sigma_{1}$ and $\Sigma_{2}$ are dual; i.e., they can be interchanged in definition (5.2). If $\Sigma_{1}$ is a Lagrangian plane, then, obviously, $\Sigma_{2}=\Sigma_{1}$.

Lemma 3. If $\Sigma_{1}$ is a vortex plane, then

(1) $\Sigma_{1} \cap \Sigma_{2}=\{0\}$;

(2) $\Sigma_{2}$ is also a vortex plane.

Indeed, let $z \in \Sigma_{1} \cap \Sigma_{2}$, and let $z \neq 0$. Then the vector $z \in \Sigma_{1}$ is skew-orthogonal to all vectors in $\Sigma_{1}$. However, this contradicts the nondegeneracy of the form $\widetilde{\omega}$. Furthermore, let $\Sigma_{2}$ be a nonvortex plane. Then there exists a nonzero vector $z \in \Sigma_{2}$ that is skew-orthogonal to all vectors in $\Sigma_{2}$. Since $\mathbb{R}^{2 n}=\Sigma_{1} \oplus \Sigma_{2}$ and since $\Sigma_{2}$ is skew-orthogonal to $\Sigma_{1}$, it follows that the vector $z$ is skew-orthogonal to all vectors in $\mathbb{R}^{2 n}$. But this contradicts the nondegeneracy of the symplectic structure $\omega$.

Theorem 9. Let $\Sigma_{1}$ and $\Sigma_{2}$ be dual vortex planes. If $\Sigma_{1}$ is an invariant plane of the Hamiltonian system, then so is $\Sigma_{2}$, and vice versa. 
Thus invariant vortex planes of linear Hamiltonian systems come in pairs. This observation generalizes the result in Sec. 2 on two solutions of the quadratic matrix equation (2.1). It can readily be shown that the vortex planes $\{\dot{x}=A x\}$ and $\left\{\dot{x}=A^{\prime} x\right\}$ are skew-orthogonal with respect to the standard symplectic structure in $\mathbb{R}^{2 n}=\left\{x_{1}, \ldots, x_{n}, y_{1}, \ldots, y_{n}\right\}$. Theorem 9 can be regarded as a symplectic analog of Viete's theorem.

Proof of Theorem 9. Let $a_{1}, \ldots, a_{n}$ and $b_{1}, \ldots, b_{n}$ be some bases in the $n$-dimensional vector spaces $\Sigma_{1}$ and $\Sigma_{2}$.

Since these spaces are skew-orthogonal, we have

$$
\left(J a_{i}, b_{j}\right)=0
$$

for all $i, j=1, \ldots, n$. Clearly,

$$
\begin{aligned}
& \Sigma_{1}=\left\{z:\left(J b_{1}, z\right)=\cdots=\left(J b_{n}, z\right)=0\right\}, \\
& \Sigma_{2}=\left\{z:\left(J a_{1}, z\right)=\cdots=\left(J a_{n}, z\right)=0\right\} .
\end{aligned}
$$

Let $\Sigma_{1}$ be an invariant plane of the linear system (5.1). Then

$$
\left(J b_{j}, z\right)^{\cdot}=\left(J b_{j}, J B z\right)=-\left(B b_{j}, z\right)=0
$$

for $z \in \Sigma_{1}$. Therefore,

or, which is the same,

$$
-B b_{j}=\sum \lambda_{j, k} J b_{k}, \quad \lambda_{k} \in \mathbb{R}
$$

$$
J B b_{j}=\sum \lambda_{j, k} b_{k} .
$$

By Lemma 3 , the vectors $a_{1}, \ldots, a_{n}, b_{1}, \ldots, b_{n}$ form a basis in $\mathbb{R}^{2 n}$. Consequently,

$$
J B a_{j}=\sum \mu_{j, s} a_{s}+\sum æ_{j, r} b_{r},
$$

whence, in view of (5.3), we obtain the relation

$$
\sum \mathfrak{j}_{j, r}\left(b_{r}, J b_{i}\right)=\left(J B a_{j}, J b_{i}\right)=-\left(a_{j}, B b_{i}\right)=\left(J a_{j}, J B b_{i}\right)=\left(J a_{j}, \sum \lambda_{i, k} b_{k}\right)=0 .
$$

It follows that

$$
\left(\sum æ_{j, r} b_{r}, J b_{i}\right)=0
$$

for all $i$. Since $\Sigma_{2}$ is a vortex plane, we have $\sum æ_{j, r} b_{r}=0$, and therefore all $æ_{j, r}$ are zero. However, in this case (5.6) will have the form (5.5). These relations mean that $\Sigma_{2}$ is an invariant plane, as desired.

Let us consider a typical case in which the linear Hamiltonian system (5.1) has a simple spectrum. Let the spectrum contain $r$ real pairs of eigenvalues, $s$ pure imaginary pairs of eigenvalues, and $f$ complex quadruples of eigenvalues. Clearly,

$$
n=s+r+2 f .
$$

Theorem 10. There exist pairs of $n$-dimensional invariant vortex planes if and only if

$$
n=2 n_{1}+4 m_{1}=2 n_{2}+4 m_{2},
$$

where $n_{j}$ and $m_{j}$ are nonnegative integers such that

$$
n_{1}+n_{2}=s+r, \quad m_{1}+m_{2}=f .
$$

The number of distinct pairs of invariant vortex planes is equal to that of distinct decompositions (5.7) of the number $n$ under the constraints (5.8).

Let us show how the invariant vortex planes in Theorem 10 can be constructed. For this, we once again use Williamson's theory of normal forms for linear Hamiltonian systems. In the case of a simple spectrum, the phase space $\mathbb{R}^{2 n}$ splits into a direct sum of skew-orthogonal 2- or 4dimensional invariant subspaces such that the Hamiltonian is represented as the sum of quadratic forms on these subspaces. These quadratic forms are partial Hamiltonians. A real simple pair of eigenvalues $\pm a$ gives rise to the partial Hamiltonian $a\left(p^{2}-q^{2}\right) / 2$, for a pure imaginary pair $\pm i b$ 
we have the partial Hamiltonian $b\left(p^{2}+q^{2}\right) / 2$, and the partial Hamiltonian corresponding to a quadruple of eigenvalues is

$$
a\left(p_{1} q_{1}+p_{2} q_{2}\right)+b\left(p_{1} q_{2}-p_{2} q_{1}\right) .
$$

To obtain the first invariant vortex plane, one should set $p=q=0$ in the expressions for the $n_{1}$ partial Hamiltonians corresponding to the real and pure imaginary pairs of eigenvalues and $p_{1}=p_{2}=q_{1}=q_{2}=0$ in the expressions for the $m_{1}$ partial Hamiltonians corresponding to the complex quadruples of eigenvalues. The remaining canonical variables will be coordinates on the desired $n$-dimensional plane. The second (dual) invariant vortex plane is constructed in a similar way with the obvious replacement of $n_{1}$ and $m_{1}$ by $n_{2}$ and $m_{2}$, respectively. The proof of the fact that there are no other invariant vortex planes is rather cumbersome, and we do not present it here.

Instead, we consider a simple example. Let $n=2$, and let the spectrum of a Hamiltonian system be a complex quadruple. In this case, either $m_{1}$ or $m_{2}$ is equal to unity. Therefore, according to (5.7), we have $n \geqslant 4$, and (by Theorem 10) the Hamiltonian system does not admit any invariant vortex planes at all.

As was already said, the Hamiltonian can be reduced to the form (5.9). A straightforward calculation shows that the corresponding Hamiltonian system has exactly two invariant planes, namely, $p_{1}=p_{2}=0$ and $q_{1}=q_{2}=0$, but both of them are Lagrangian.

\section{References}

1. V. V. Kozlov, General Theory of Vortices, Springer-Verlag, 2003.

2. J. Williamson, "On an algebraic problem concerning the normal forms of linear dynamical systems," Amer. J. Math., 58, No. 1, 141-163 (1936).

3. F. R. Gantmakher, Theory of Matrices [in Russian], Nauka, Moscow, 1967.

4. V. V. Kozlov and A. A. Karapetyan, "On the stability degree," Differentsial'nye Uravneniya, 41, No. 2, 186-192 (2005); English transl.: Differential Equations, 41, No. 2, 195-201 (2005).

5. V. V. Kozlov, "Linear systems with a quadratic integral," Prikl. Mat. Mekh., 56, No. 6, 900-906 (1992); English transl.: J. Appl. Math. Mech., 56, No. 6, 803-809 (1992).

6. S. I. Gelfand, "On the number of solutions of a quadratic equation," In: GLOBUS, IUM General Seminar [in Russian], No. 1, MCCME, Moscow, 2004, pp. 124-133.

7. V. V. Kozlov, "The spectrum of a linear Hamiltonian system and the symplectic geometry of the complex Artin space," Dokl. Ross. Akad. Nauk, 393, No. 24, 453-455 (2003).

V. A. Steklov Institute of Mathematics

e-mail: kozlov@pran.ru

Translated by V. M. Volosov 